\title{
Suction Procedure
}

National Cancer Institute

\section{Source}

National Cancer Institute. Suction Procedure. NCI Thesaurus. Code C51939.

A procedure that makes use of a vacuum for the removal of fluid or tissue. 